\title{
Reliability and Validity of "L" Test in Young Elderly
}

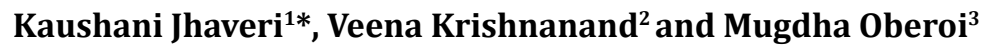

${ }^{1}$ Physiotherapist, New Horizons Group, Maharashtra, India

${ }^{2}$ Former Professor and Head of Department, K.J. Somaiya College of Physiotherapy, Director of Women Empowerment and Child Care Foundation, Maharashtra, India

${ }^{3}$ Assistant Professor, K.J. Somaiya College of Physiotherapy, Maharashtra, India

*Corresponding Author: Kaushani Jhaveri, Physiotherapist, New Horizons Group, Maharashtra, India.

Received: August 29, 2019; Published: September 19, 2019

DOI: 10.31080/ASOR.2019.02.0103

\begin{abstract}
Background: The ability to walk at home and outside safely is essential for all adults. Functional mobility, balance, anticipatory and reactive control may get hampered or altered with ageing. Various intrinsic factors such as strength of muscles, joint mobility and extrinsic factors like uneven ground, obstacles, public transports can affect balance. "L" test is a modified version of timed up and go test. "L" test is a newer outcome measure. Therefore, it is essential to establish normative values which can be used for reference for future studies as well as for screening and management for the elderly population.

Methods: 50 asymptomatic healthy young elderly falling within the inclusion criteria were assessed using "L" Test where the subjects were asked to rise from the chair, walk along the marked path that is in the shape of "L" that is 3 meters straight, right turn and then 7 meters straight and return along the same path and sit. The time taken to complete the test was noted. After an hour, the subjects were made to repeat the test. The readings of intra-rater and inter-rater were recorded. The subjects were made to perform Timed Up and Go (TUG) Test and the readings were documented.

Result and Analysis: Reliability and Validity of “L” Test was analysed using Intra-class correlation (ICC) and Spearman's correlation. Intra-class Correlation(ICC) at 95\% Confidence Interval(CI) for intra-rater reliability is 0.822 and ICC at 95\% CI for inter-rater reliability is 0.820 . A significant positive correlation was seen between duration of "L" Test and duration of TUG $(r=0.813, p<0.001)$. Conclusion: The results of the study suggest that "L" Test has a good intra and inter rater reliability and concurrent validity with TUG Test. Therefore, it could be used as a tool for geriatric assessment of balance, function, anticipatory and reactive control as well as risk of falls in young elderly population in an urban setting.

Keywords: "L" Test; TUG; Geriatric Assessment; Physical Activity and Function; Balance; Anticipatory Control; Reactive Control; Reliability; Validity
\end{abstract}

\section{Introduction}

Ageing is defined as an age dependent or age progressive decline in intrinsic physiological function. Thereby, leading to an increase in age specific mortality rate and a decrease in age specific reproductive rate [1].

At the biological level, ageing results from the impact of the accumulation of a wide variety of molecular and cellular damage over time. This leads to a gradual decrease in physical and mental capacity and a growing risk of disease. But these changes are neither linear nor consistent, and they are only loosely associated with a person's age in years [2].

As per the census of 2011, total population of India was 1210.9 million, out of which 103.9 million were above the age of 60 . Hence, the percentage of the Elderly in total population was 8.6\% [3]. 
The number of individuals over 60 years is growing faster than any other age group, throughout the world. This number was 900 million in 2015 and is estimated to grow to almost two billion by 2050 [4].

The ability to walk at home and outside safely is critical for all humans. It provides the freedom of movement to an individual at home and in different environments.

Functional mobility is the manner in which people are able to move around in the environment in order to participate in the activities of daily living and, move from one place to another. Balance is the ability to stay upright in coordination with body movements for smooth and effective functional mobility. Anticipatory and reactive control during movement, is defined as the ability to make postural adjustments prior to accomplishing a task.

These abilities may be affected during ageing due to some of the intrinsic factors such as strength of muscles, joint mobility, vision, hearing, proprioception, sensations etc. The extrinsic factors like uneven ground, obstacles, public transport, dark spaces and crowded areas add to the difficulty in carrying out various activities.

Various tests are being used to test balance and coordination in routine clinical practice. "L" test, is a modified version of Timed Up and Go(TUG) Test. It is a recent addition to these evaluation procedures.

The "L" test is designed in such a way, that it does not have a straight path of the movement. It has turns that simulate home or/ and outdoor environment. This test is used to assess balance, functional mobility and anticipatory changes. In addition, it helps in screening elderly who are at a risk of falls.

"L" Test is a quick method of geriatric assessment of balance as well as the function as compared to other tests like Bergs Balance Scale, Dynamic Gait Index and was designed to evaluate advanced functional ambulation, including turning in both directions and walking for relatively long distance, as this is not covered by popular functional measures, such as the TUG Test $[5,6]$.

The reliability of "L" test for Indian population is yet to be carried out. The normative values for the elderly population need to be ascertained, as well. These values will act as the reference for future studies as well as the planning the management of the elderly in the community, in India.

\section{Methodology}

This Cross-sectional Study consisted of 50 community dwelling young elderly between the age group of 60-70 years. The partici- pants were selected for the study using convenient sampling technique.

The participants included in the study were asymptomatic healthy individuals with or without co-morbidities (Diabetes Mellitus or Hypertension but levels under control), corrected vision and hearing, independent ambulation with or without aid and a Mini Mental State Examination(MMSE) Scale Score of 27 and above [7]. Any individual who had undergone any major recent surgery (within past 3 months), any major orthopedic condition, any neurological deficits, any cardiorespiratory involvement or any exercising individuals were excluded.

\section{Procedure}

229 subjects were screened to rule out exclusion criteria. The 50 subjects satisfying inclusion criteria were enrolled in the study.

These individuals were explained about the study and a written consent was taken for the same. The demonstration of the test was shown, and the subjects were given a trial before beginning the test.

The participants were asked to rise from the chair, walk along the marked path that is in the shape of "L" 3 meters straight, take a right turn and then walk straight for 7 meters and return back along the same path and sit down on the chair. The time taken to complete the test was noted. The subjects were made to repeat the test after an hour by the same investigator and co-investigator respectively. The subjects were made to perform a TUG Test as well and the readings were recorded.6 The documented readings were analyzed for the intra-rater and inter-rater reliability.

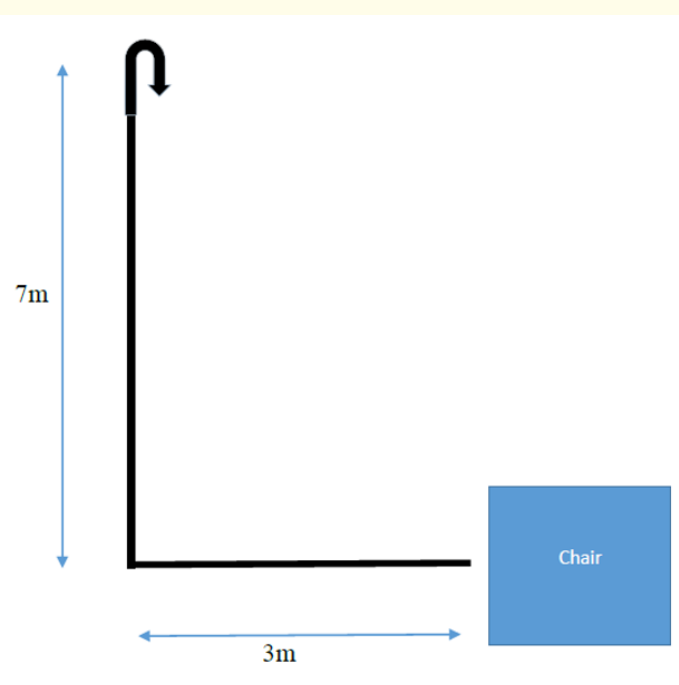

Figure 1 


\section{Results and Analysis}

The data obtained was analyzed using Statistical Package for the Statistical Sciences(SPSS) Software version 24. The data was represented on Bland-Altman Plot using Graph Pad Prism version 7.

\begin{tabular}{|l|c|c|}
\hline & Mean & SD \\
\hline “L” Test & 23.91 seconds & 2.58 seconds \\
\hline TUG & 10.78 seconds & 1.42 seconds \\
\hline
\end{tabular}

Table 1: Demographics.

\begin{tabular}{|l|c|}
\hline The intra class correlation coefficient, ICC $[\alpha]$ & $\mathrm{p}$ Value \\
\hline 0.822 [95\%CI $0.707-0.895]$ & $<0.001$ \\
\hline
\end{tabular}

Table 2: Intra Rater Reliability.

\begin{tabular}{|l|c|}
\hline The intra class correlation coefficient, ICC $[\alpha]$ & $p$ Value \\
\hline $0.820[95 \%$ CI $0.704-0.893]$ & $<0.001$ \\
\hline
\end{tabular}

Table 3: Inter Rater Reliability.

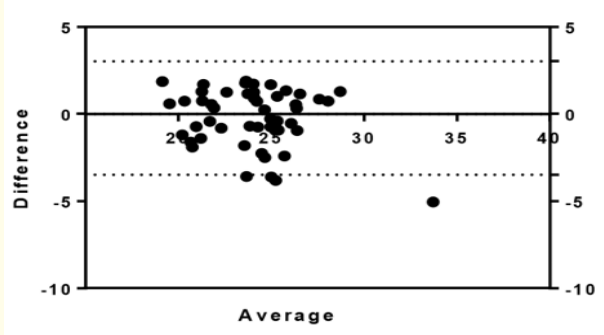

Graph 1: B land-A Itm an of L1 and L1A.

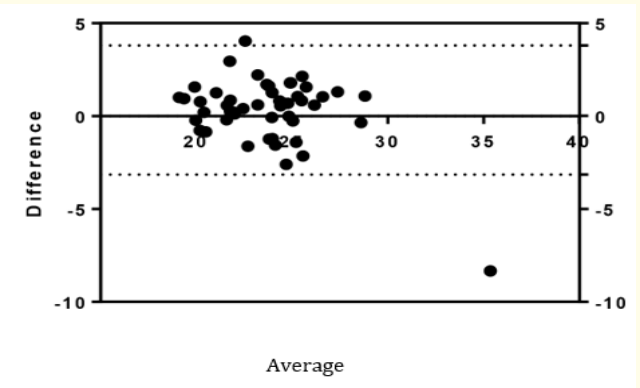

Graph 2: B lan d-A ltm an of L1 and L2.
Standard Error of Measurement(SEM) and Minimally Detectable Change(MDC) were calculated as follows:

$$
\begin{aligned}
& \mathrm{SEM}=\mathrm{SD} \times \sqrt{(1-r)} \\
& \mathrm{MDC}=1.96 \times \mathrm{SEM} \times \sqrt{2}
\end{aligned}
$$

SEM is the error that is considered as measurement error. MDC is a statistical estimate of the smallest amount of change that can be detected by a measure that corresponds to a noticeable change in ability [8].

MDC helps clinicians understand the functional changes in the clients in clinical settings; thus enabling them to interpret the outcomes in the literature by comparing statistical significance with meaningful clinical change [9].

The Standard Error of Measurement was found to be 1.088. The Minimally Detectable Change was 3.015 seconds.

\section{Concurrent validity}

A Significant Positive Correlation was achieved between the durations of " $\mathrm{L}$ " Test and durations of TUG ( $\mathrm{r}=0.813, \mathrm{p}<0.001)$ using Spearman's Rho.

\section{Discussion}

229 subjects were screened to rule out exclusion criteria. The 50 subjects satisfying inclusion criteria were enrolled in the study. The data was represented using Bland Altman Plots. In both the Intra- and Inter-rater plots, the data points close to the Bland-Altman zero line indicate that not much a variation is seen in the readings.

The results of this study indicate good intra $(r=0.822)$ and inter rater reliability $(r=0.820)$ for the above mentioned population. A positive correlation to confirm concurrent validity was found between "L" Test and TUG in this study.

The MDC value was 3.015 seconds. The individuals having deviation of greater or lesser than this value may experience difficulty or improved ability respectively in maintaining balance during physical activity and function. Therefore, this value would help in predicting a true change in an elderly person.

J.S. Kim., et al. in their study on chronic stroke patients, found a significant positive correlation between the duration of "L" Test 
and duration of TUG test $(r=0.89, p<0.001)$. The findings of the current study too, concur with the results of the said study [5].

A comparative study on the hospitalized frail elderly by Nguyen VC., et al. high interrater reliability ICC of $1.00,95 \%$ confidence interval, [0.99-1.00] and intra rater reliability ICC of $0.97,95 \%$ confidence interval, [0.95- 0.98] was found in "L" test. In addition, there was a positive correlation between the L test and TUG ( $\mathrm{r}$ $=0.96 ; p<0.001$ ). The results of the current study are similar the results of Nguyen VC., et al. [10].

The results of the study conducted by Deathe B., et al. indicated, excellent intra-rater reliability ( $\mathrm{r}=0.97$ with $95 \% \mathrm{CI}=0.93-0.98)$. Inter-rater reliability ( $\mathrm{r}=0.96$ with $95 \% \mathrm{CI}=0.94-0.97)$ was also found to be similar compared to the current study [11]

In a similar study performed by Ann Medley., et al. conducted a strong significant correlation $(\mathrm{r}=0.94, \mathrm{p}<0.001)$ was found between "L" Test and TUG [12]. The said results are in agreement of the current study, where the correlation was found to be 0.813 , between TUG and "L" test.

Thus, a good intra and inter rater reliability and concurrent validity of "L" Test of the current study suggest that "L" Test can be used as a clinical tool for evaluation of balance in the elderly individuals.

\section{Conclusion}

The results of the study suggest that "L" Test has a good intra and inter rater reliability and concurrent validity with TUG Test. Therefore, it can be used as a tool for assessment of balance, functional mobility, anticipatory and reactive control as well as risk of falls in young elderly population in an urban setting.

\section{Clinical Implications}

The "L" Test can be used to assess risk of falls as well as help in early screening for fallers, in a routine clinical setting. It is also useful in assessing balance as well as anticipatory and reactive control in elderly. This test is a quicker and faster tool for geriatric assessment.

\section{Bibliography}

1. Flatt T. "A new definition of aging?" Frontier in Genetics 3 (2012): 148

2. http://www.who.int/mediacentre/factsheets/fs404/en/

3. http://mospi.nic.in/sites/default/files/publication_reports/ ElderlyinIndia_2016.pdf
4. http://www.who.int/entity/mediacentre/factsheets/fs404/ en/

5. JS Kim., et al. "Reliability and validity of L test in Participants with Chronic Stroke”. Physiotherapy 101 (2015): 161-165.

6. Podsiadlo D and Richardson S. "The Time "Up and Go": A Test of Basic Functional Mobility for Frail Elderly Persons". Journal of the American Geriatrics Society 39.2 (1991): 142-148.

7. Folstein MF., et al. "'Mini-mental state': a practical method for grading the cognitive state of patients for the clinician". Journal of Psychiatric Research 12 (1975): 189-198.

8. www.rehabmeasures.org.

9. Vimonwan Hiengkaew., et al. "Minimal Detectable Changes of the Berg Balance Scale, Fugl-Meyer Assessment Scale, Timed "Up and Go" Test, Gait Speeds, and 2-Minute Walk Test in Individuals with Chronic Stroke with Different Degrees of Ankle Plantarflexor Tone". Archives of Physical Medicine and Rehabilitation (2012): 93.

10. Nguyen VC., et al. "Measurement properties of $\mathrm{L}$ test for gait in hospitalized elderly". American Journal of Physical Medicine and Rehabilitation 86 (2007): 463-468.

11. Deathe AB and Miller WC. "The L Test of functional mobility: Measurement Properties of a modified version of the "timed up and go" test designed for people with lower limb amputation". Physical Therapy 86 (2005): 1641-1647.

12. Ann Medley., et al. "Contribution of Age and Balance Confidence (ABC) to Functional Mobility Test Performance: Diagnostic Accuracy of "L" Test and Normal-Paced Timed Up and Go". Journal of Geriatric Physical Therapy 86 (1979): 420-428.

\section{Volume 2 Issue 10 October 2019}

(c) All rights are reserved by Kaushani Jhaveri., et al. 